\title{
Requirements and Technology Advances for Global Wind Measurement with a Coherent Lidar: A Shrinking Gap
}

\author{
Michael J. Kavaya, Jirong Yu, Grady J. Koch, \\ Farzin Amzajerdian, Upendra N. Singh \\ NASA Langley Research Center \\ michael.j.kavaya@nasa.gov
}

G. David Emmitt

Simpson Weather Associates

SPIE Lidar Remote Sensing for Environmental Monitoring VIII San Diego, CA

Aug. 26-30, 2007 


\section{Customers for Global Winds}

\begin{tabular}{|c|c|c|}
\hline Air Force & 2007 & $\begin{array}{l}\text { "Among the } 15 \text { missions recommended by the National Research Council } \\
\text { Decadal Survey Report on Earth Science and Applications from Space, the measurement of } \\
\text { global tropospheric winds would provide the greatest potential benefit to the mission of the } \\
\text { United States Air Force. DWL would provide data critical to the DoD's need to characterize } \\
\text { and exploit the environment for strategic and operational advantage." Aug. 1, } 2007 \text { Letter } \\
\text { from Director of Weather to NASA SMD AA (Dr. Alan Stern) }\end{array}$ \\
\hline NRC & 2007 & $\begin{array}{l}\text { One of } 15 \text { recommended NASA earth science missions. [National Research Council (NRC), } \\
\text { "Earth Science and Applications from Space: National Imperatives for the Next Decade and } \\
\text { Beyond," p. 2-14, The National Academies Press, Wash DC, "Decadal Survey" (DS)] }\end{array}$ \\
\hline NASA & 2007 & $\begin{array}{c}\text { Highest priority of "Weather" Science Focus Area. [NASA, "Science Plan for NASA's } \\
\text { Science Mission Directorate," pp. 60-61] }\end{array}$ \\
\hline $\begin{array}{l}\text { US/NSTC/ } \\
\text { CENR/ } \\
\text { IWGEO } \\
\text { (nOW GEO) }\end{array}$ & 2005 & $\begin{array}{l}\text { Highest priority for improved weather forecasting. High importance to Weather, Disasters, } \\
\text { Oceans, Climate, and Human Health Societal Benefit Areas; medium importance to } \\
\text { Agriculture and Ecology. [National Science and Technology Council, Committee on } \\
\text { Environment and Natural Resources, Interagency Working Group on Earth Observations, } \\
\text { "Strategic Plan for the U.S. Integrated Earth Observation System," p. 37] }\end{array}$ \\
\hline ESA & 1999 & $\begin{array}{l}\text { "improvement in analyzing global climate, its variability, predictability and change requires } \\
\text { measurements of winds throughout the atmosphere." [ESA, "Reports for Mission Selection. } \\
\text { The Four Candidate Earth Explorer Core Missions. Atmospheric Dynamics Mission," SP-1233 } \\
\text { (4)] }\end{array}$ \\
\hline $\begin{array}{c}\text { NPOESS } \\
\text { (IPO) }\end{array}$ & 1996 & $\begin{array}{c}\text { Highest priority unaccommodated NPOESS measurement. "Direct tropospheric wind } \\
\text { measurements would provide a greater impact on numerical weather prediction than any } \\
\text { other new space-based observation." [NPOESS/IPO (Integrated Program Office), } \\
\text { "Unaccommodated Environmental Data Records: Technology Status and Promising } \\
\text { Technological Areas," pp. 4, 6] }\end{array}$ \\
\hline WMO & 1996 & $\begin{array}{c}\text { "Observations of upper winds are essential for operational weather forecasting on all scales } \\
\text { and at all latitudes, ..." [WMO, "Guide to Meteorological Instruments and Methods of } \\
\text { Observation," } 6^{\text {th }} \text { ed., WMO-No. 8, pp. I.12-31, I.13-1] }\end{array}$ \\
\hline
\end{tabular}




\section{Early Mission Concept}

- $525 \mathrm{~km}$ orbit height

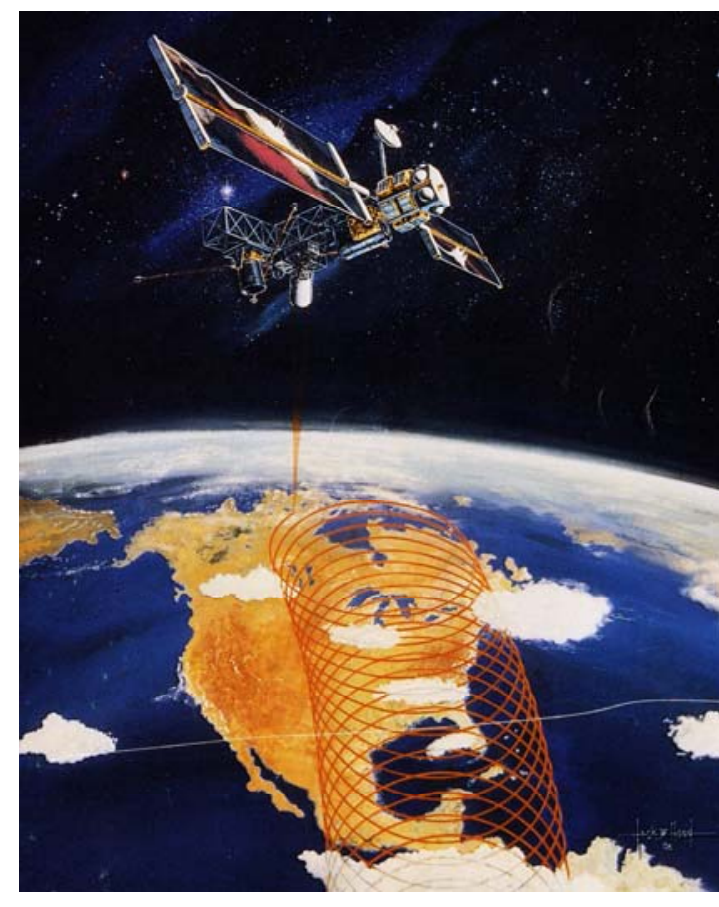

- Single pulsed Doppler lidar system covers troposphere

- Continuously rotating telescope/scanner

- Line of sight (LOS) wind profiles from each laser shot

- $20 \mathrm{~J}$ pulse energy

$\bullet \sim 1.5 \mathrm{~m}$ rotating telescope

- Requirement: eyesafe solid state pulsed laser

- 2-micron technology for coherent detection

- $20 \mathrm{~mJ} / 5 \mathrm{~Hz}$ demonstrated vs. $20 \mathrm{~J} / 10 \mathrm{~Hz}$ required

- Energy deficiency of x1000 (30 dB)! 


\section{Ideas, Advances, and Changes}

\begin{tabular}{|l|l|}
\hline \multicolumn{1}{|c|}{ Factor } & \multicolumn{1}{c|}{ Notes } \\
\hline 2-Micron Laser Technology Advances & $\begin{array}{l}\text { Pulse energy, pulse repetition } \\
\text { frequency (PRF) }\end{array}$ \\
\hline Hybrid Doppler Lidar Concept & $\begin{array}{l}\text { Use both coherent and direct detection } \\
\text { Doppler lidars to cover the troposphere }\end{array}$ \\
\hline $\begin{array}{l}\text { Step-Stare Scanning and Shot } \\
\text { Accumulation }\end{array}$ & $\begin{array}{l}\text { Permit multiple laser shots to be } \\
\text { combined for each LOS wind profile } \\
\text { measurement }\end{array}$ \\
\hline Lower Orbit Height to $400 \mathrm{~km}$ & $\begin{array}{l}\text { SNR proportional to inverse range } \\
\text { squared }\end{array}$ \\
\hline $\begin{array}{l}\text { Demonstration Wind Mission } \\
\text { Requirements }\end{array}$ & $\begin{array}{l}\text { Relaxed measurement requirements for } \\
\text { a demonstration mission }\end{array}$ \\
\hline
\end{tabular}




\section{2-Micron Laser Advances at LaRC}

\begin{tabular}{|c|c|c|}
\hline Category & Advancement & $\begin{array}{c}\text { Current Space } \\
\text { Requirement }\end{array}$ \\
\hline Pulse Energy & $0.02 \mathrm{~J}$ to $1.2 \mathrm{~J}(\mathrm{x} 60)$ & $0.25 \mathrm{~J}$ \\
\hline $\begin{array}{c}\text { Pulse Repetition } \\
\text { Frequency }\end{array}$ & $1 \mathrm{~Hz}$ to $10 \mathrm{~Hz}$ & $5 \mathrm{~Hz}$ \\
\hline $\begin{array}{c}\text { Wallplug Electrical } \\
\text { Efficiency }\end{array}$ & Invented Ho:Tm:LuLiF (+20\% improvement) & $\sim 1.4 \%$ \\
\hline Host Crystal & YAG to YLF to LuLiF & LuLiF \\
\hline Components & Oscillator, Preamplifier, Amplifiers & Oscillator \& 1 Amplifier \\
\hline Mode & Normal to Q-Switched to Injection Seeded & Injection Seeded \\
\hline Beam Quality & 1.1 x Diffraction Limit & 1.1 x Diffraction Limit \\
\hline Pulse Spectrum & $\sim 2.5$ MHz FWHM & $\sim 2.5$ MHz FWHM \\
\hline $\begin{array}{c}\text { Cooling Method } \\
\text { All Liquid to Half Conductive, Half Liquid to } \\
\text { All Conductively Cooled }\end{array}$ & $\begin{array}{c}\text { All Conductively } \\
\text { Cooled }\end{array}$ \\
\hline $\begin{array}{c}\text { Pump Diode Array } \\
\text { Package }\end{array}$ & C to A to AA to G & G package \\
\hline
\end{tabular}




\section{2-Micron Laser Advances at LaRC}

Oscillator

\&

Amplifier Designs

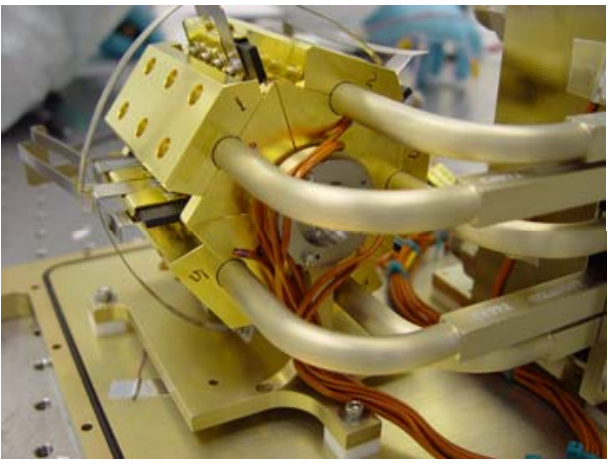

Fully conductively cooled $(11 / 03)$

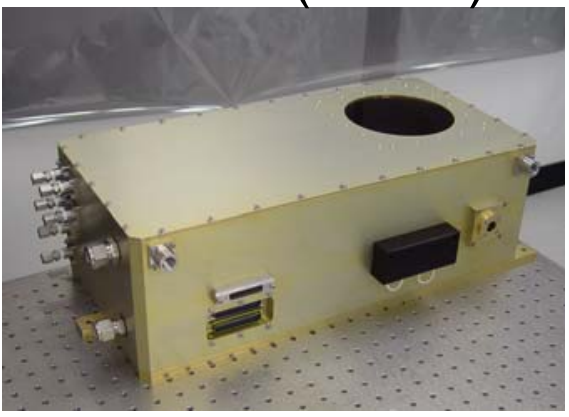

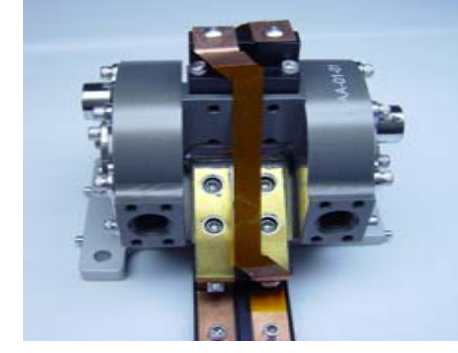

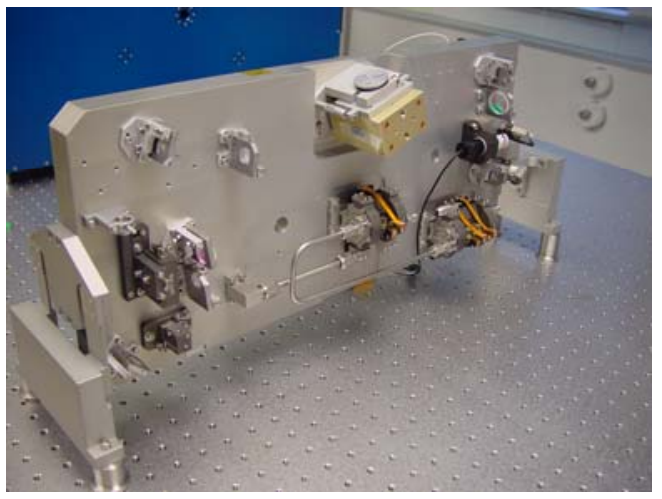

Engineered Packaging

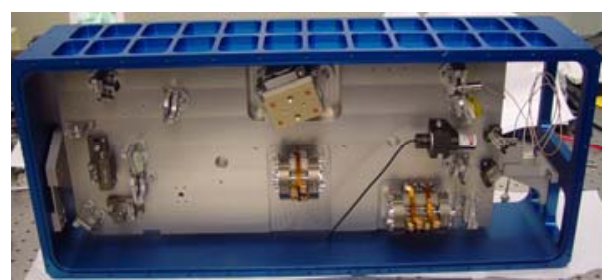

One-Joule Pulse Output Result

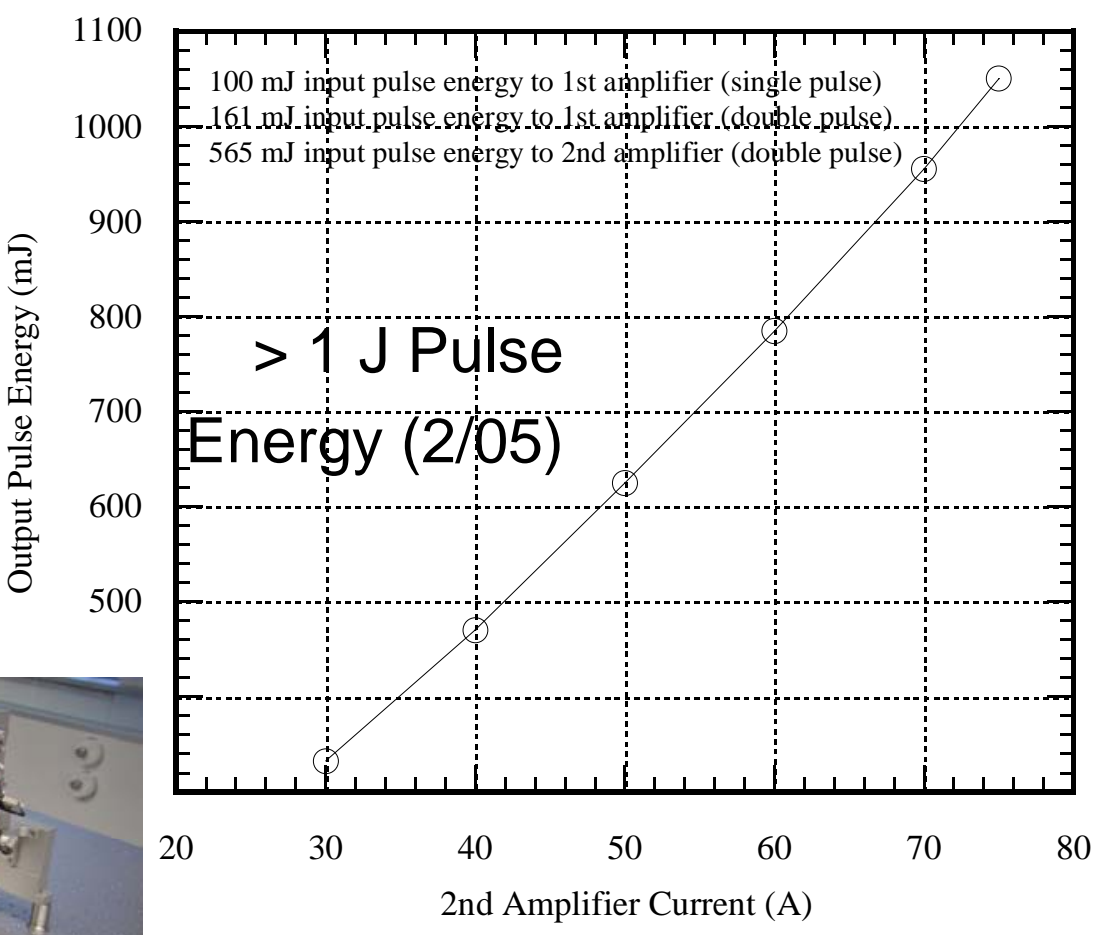

Also:

- Temperature control

- New LDA packages

- $1 \mathrm{~J}$ w/o preamplifier (12/05)

- Pulse spectrum

- Beam quality

- Efficiency 
Complementary Lidars Together Lower Total Mass, Power, Cost, Risk

Green represents percentage of sampled volumes when coherent subsystem provides the most accurate LOS measurement; Yellow is for direct detection; Gray is when neither system provides an observation that meets data requirements

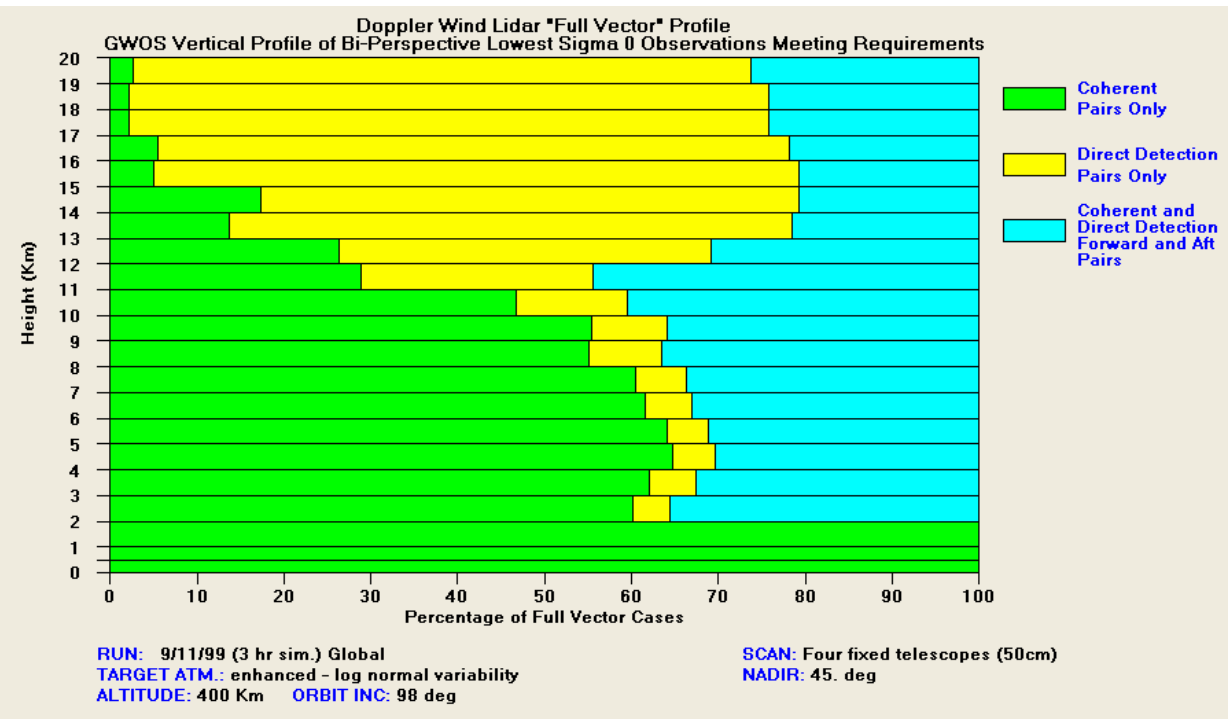

GWOS with enhanced aerosol mode

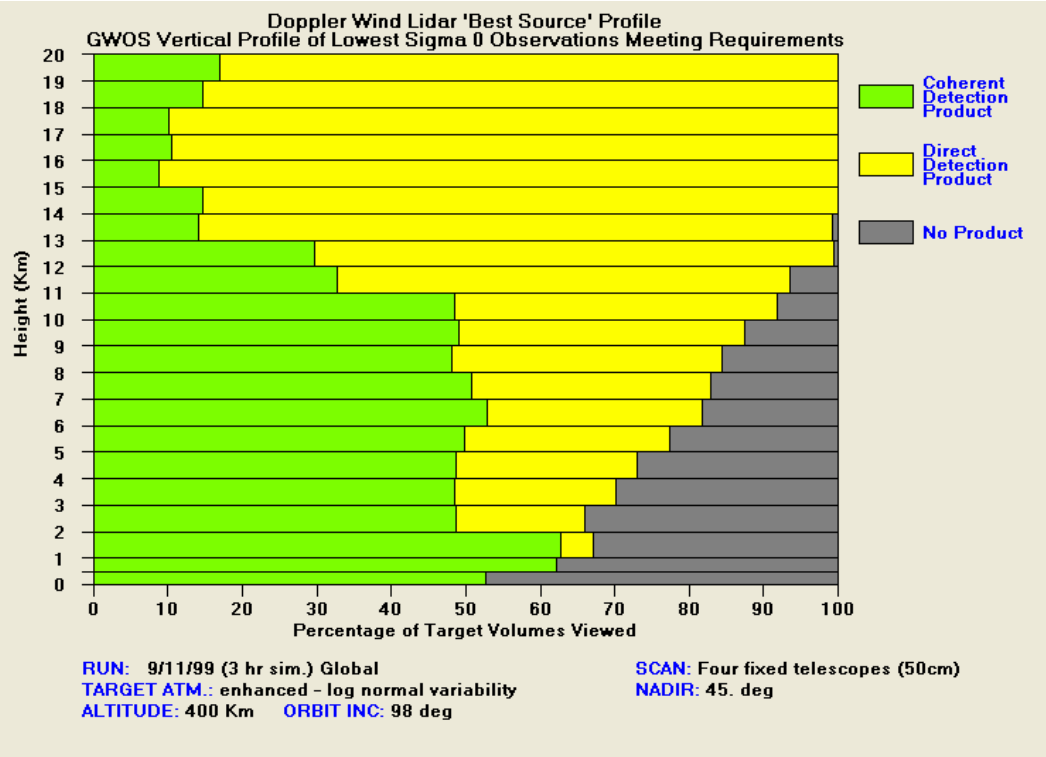

When two perspectives are possible Green: both perspectives from coherent system Yellow: both perspectives from direct molecular Blue: one perspective coherent, one perspective direct 


\section{Hybrid Doppler Lidar Meets Requirements GWOS with background aerosol mode}

Doppler Wind Lidar Data Quality Profile
Observation Uncertainty : HLOS : Clouds : w/ False Alarms

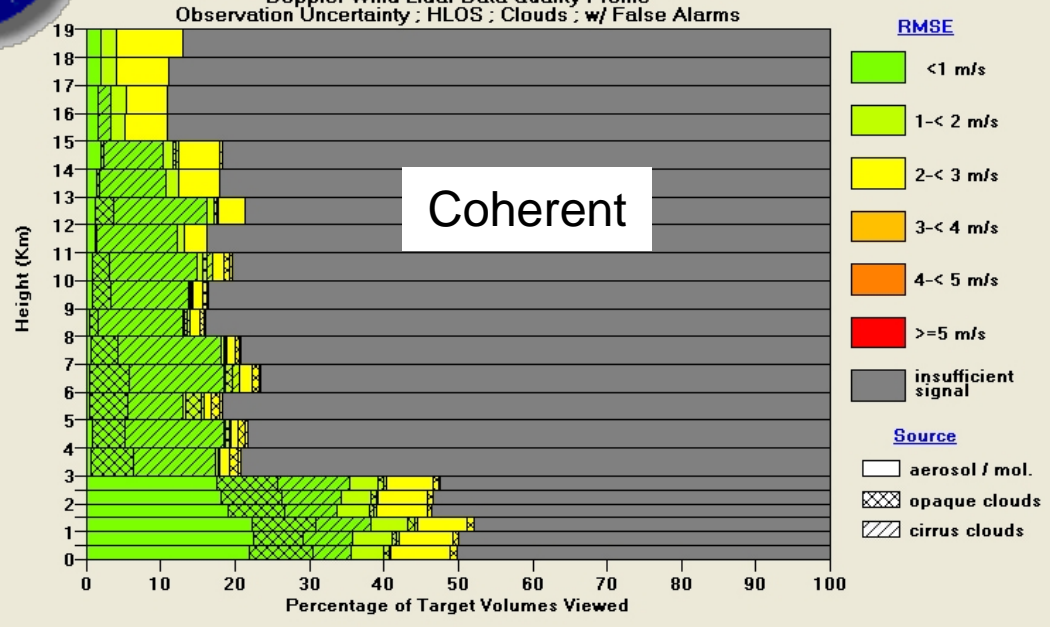

RUN: 9/28/1999 $0000 \mathrm{Z}(3 \mathrm{hr}$ sim.) Global TARGET ATM.: background- log normal variability ALTITUDE: $400 \mathrm{Km}$.

DETECTION: Coherent SCAN: 4 pt. Step_Stare WAVELENGTH: $2.052 \mathrm{um}$ NADIR: 45. deg
ENERGY: $0.25 \mathrm{~J}$ APERTURE: $0.5 \mathrm{~m}$ PLT PWR: 125. W ENERGY: $0.25 \mathrm{~J}$ APERTURE: $0.5 \mathrm{~m}$ PLT PWR: 125.
PRF: $5 . \mathrm{Hz}$ ACCUM. TIME: $12 \mathrm{~s}$ BETA THR.: 50.\%

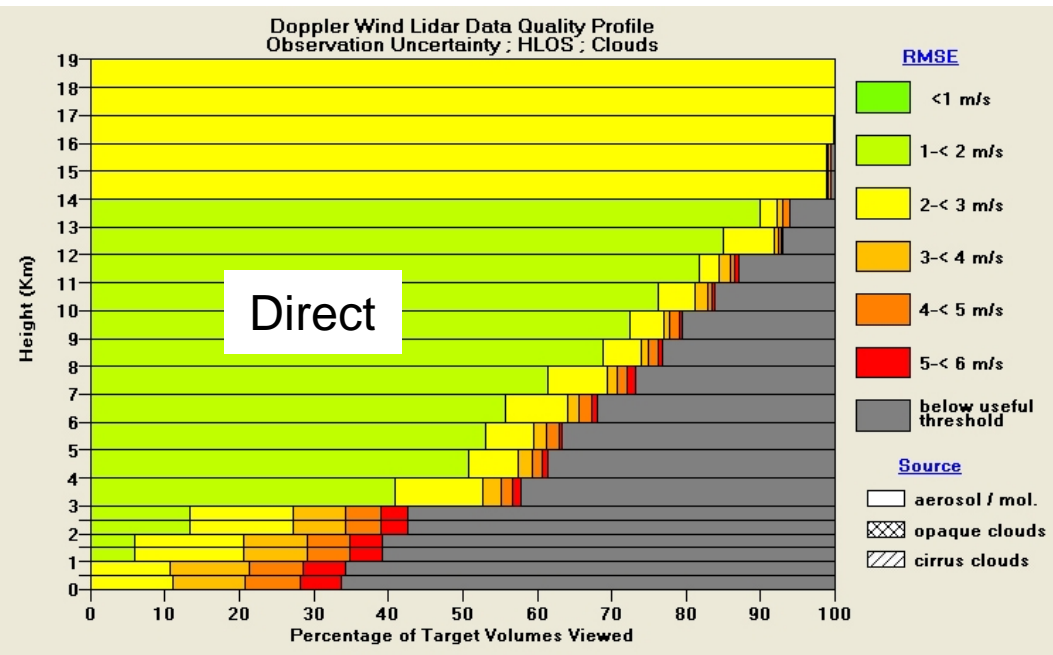

RUN: $9 / 28 / 19990000 \mathrm{Z}(3 \mathrm{hr}$ sim.) Global TARGET ATM.: background- log normal variabil ALTITUDE: $400 \mathrm{Km}$ ORBIT INC: $98 \mathrm{deg}$

DETECTION: Double-edge mol. SCAN: 4 pt. Step_Stare

WAVELENGTH: $0.355 \mathrm{um}$ NADIR: 45. deg

ENERGY at 0.355 um: $0.36 \mathrm{~J}$ APERTURE: $0.5 \mathrm{~m}$

\section{$0.25 \mathrm{~J}, 5 \mathrm{~Hz}, 0.5 \mathrm{~m}$ GWOS with enhanced aerosol mode $1 / 0.3 \mathrm{~J}, 100 \mathrm{~Hz}, 0.5 \mathrm{~m}$}

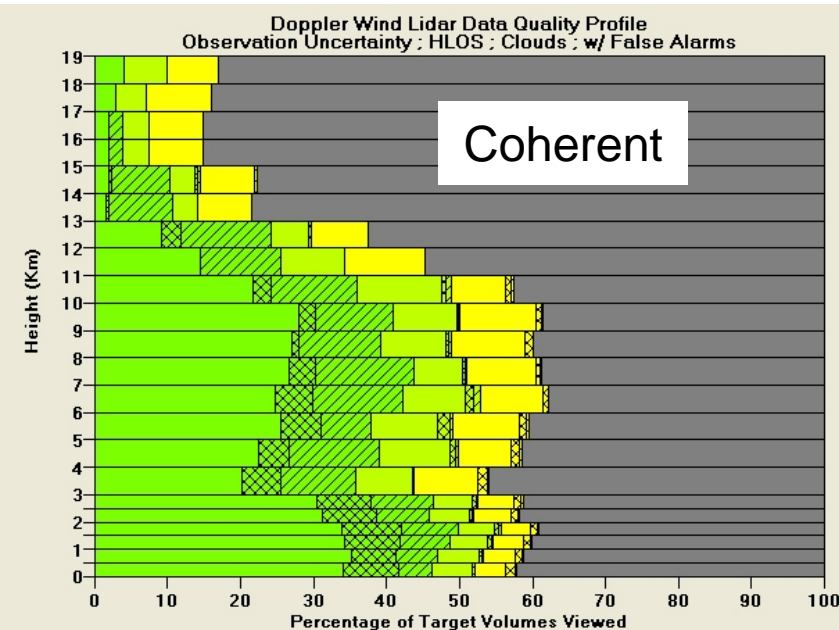

RUN: 9/28/1999 $0000 \mathrm{Z}(3 \mathrm{hr} \mathrm{sim}$.) Global TARGET ATM.: enhanced- log normal variabil VERTICAL INT. >PBL: $1 . \mathrm{Km} \quad<=$ PBL: $0.5 \mathrm{Km}$
DETECTION: Coherent SCAN: 4 pt. Step_Stare ENEREY 0.25 . APERTURE: 0.5 . 45. deg ENERGY: $0.25 \mathrm{~J}$ APERTURE: $0.5 \mathrm{~m}$ PLT PWR: 125.
PRF: $5 . \mathrm{Hz}$ ACCUM. TIME: $12 \mathrm{~s}$ BETA THR.: 50.\%

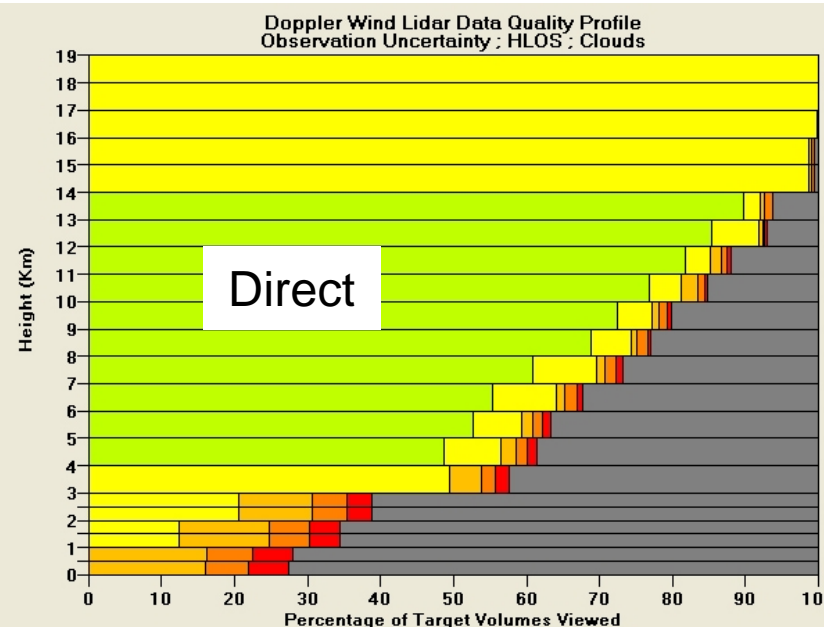

RMSE

RMSE

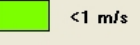

$\square 1-<2 \mathrm{~m} / \mathrm{s}$

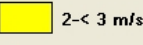

$\square-<4 \mathrm{~m} / \mathrm{s}$

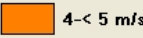

$>=5 \mathrm{~m} / \mathrm{s}$

insufficient $_{\text {signal }}$

Source

aerosol $/ \mathrm{mol}$.

RUN: 9/28/1999 $0000 \mathrm{Z}(3 \mathrm{hr}$ sim.) Global
TARGET ATM.: enhanced- log normal variabilty

TARGET ATM.: enhanced- log normal variability
ALTITUDE: $400 \mathrm{Km}$ ORBIT INC: 98 deg VERTICAL INT. $>$ PBL: $1 . \mathrm{Km} \quad<=$ PBL: $0.5 \mathrm{Km}$

DETECTION: Double-edge mol SCAN-4 pt Step Store WAVELENGTH: 0.355 um NADIR: $45 . \mathrm{deg}$ ENERGY at 0.355 um: $0.36 \mathrm{~J}$ APERTURE: $0.5 \mathrm{~m}$
PRF: $100 . \mathrm{Hz}$ ACCUM. TIME: $12 \mathrm{~s}$ PLT PWR: 824.742 


\section{AsA Step-Stare Scanning \& Shot Accumulation}
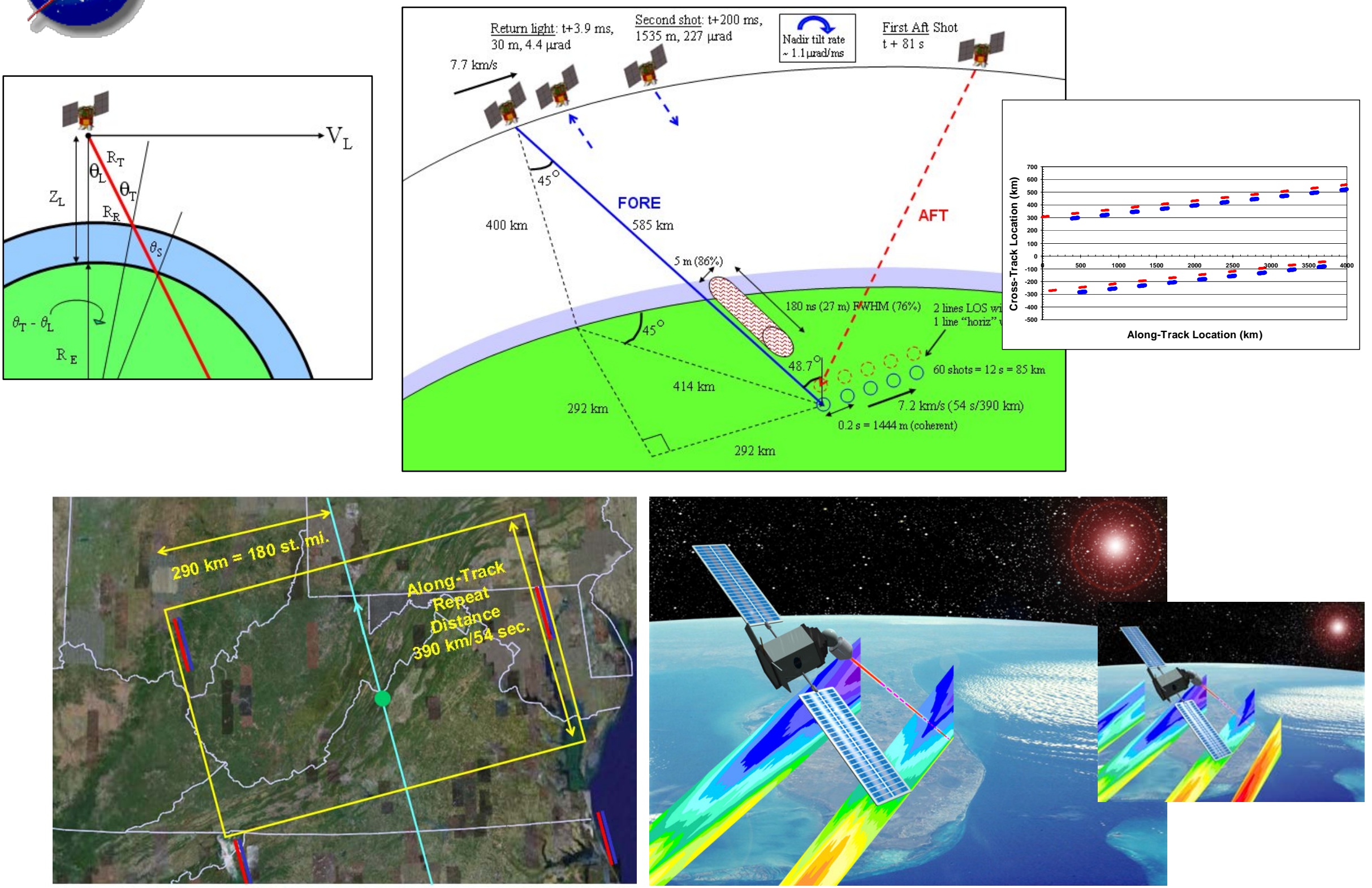


\section{Step-Stare Scanning \& Shot Accumulation}

GWOS:

- 12 second shot accumulation/azimuth angle

- Ground spot moves forward by $85 \mathrm{~km}$

- Coherent lidar at $5 \mathrm{~Hz}$

- 60 shot attempted accumulation (clouds may block some)

- If 60 successful, lower pulse energy by $87 \%$ 


\section{Lower Orbit Height}

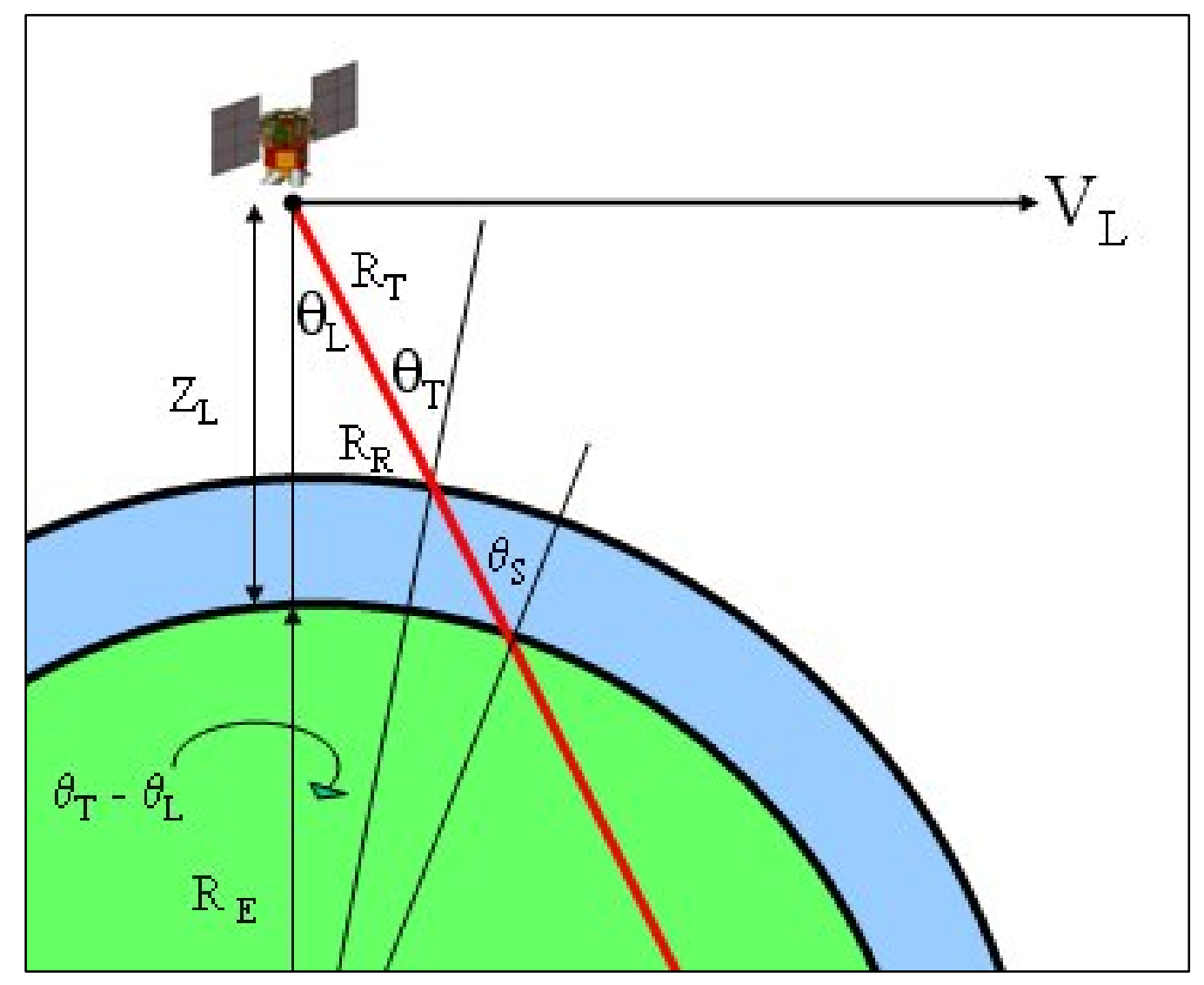

GWOS:

$\theta_{\mathrm{L}}=45$ degrees

$\mathrm{Z}_{\mathrm{L}}: 525 \rightarrow 400 \mathrm{~km}$

$\mathrm{R}_{\mathrm{T}}: 776 \rightarrow 585 \mathrm{~km}$

SNR + $2.5 \mathrm{~dB}$ 


\section{Demonstration Requirements}

\begin{tabular}{|c|c|c|c|c|}
\hline & Demo & Threshold & Objective & \\
\hline Vertical depth of regard (DOR) & $0-20$ & $0-20$ & $0-30$ & $\mathrm{~km}$ \\
\hline $\begin{array}{l}\text { Vertical resolution: } \\
\text { Tropopause to top of DOR } \\
\text { Top of BL to tropopause }(\sim 12 \mathrm{~km}) \\
\text { Surface to top of BL }(\sim 2 \mathrm{~km})\end{array}$ & $\begin{array}{l}2 \\
2 \\
1\end{array}$ & $\begin{array}{c}\text { Not Req. } \\
1 \\
0.5\end{array}$ & $\begin{array}{c}2 \\
0.5 \\
0.25\end{array}$ & $\begin{array}{l}\mathrm{km} \\
\mathrm{km} \\
\mathrm{km}\end{array}$ \\
\hline $\begin{array}{l}\text { Number of collocated LOS wind measurements for } \\
\text { horiz }{ }^{\mathrm{A}} \text { wind calculation }\end{array}$ & $2=$ pair & $2=$ pair & $2=$ pair & - \\
\hline Horizontal resolution ${ }^{\mathrm{A}}$ & 350 & 350 & 100 & $\mathrm{~km}$ \\
\hline Minimum Number of horizontal ${ }^{\mathrm{A}}$ wind tracks ${ }^{\mathrm{B}}$ & 2 & 4 & 12 & - \\
\hline $\begin{array}{ll}\text { Velocity error }^{\mathrm{C}} & \text { Above BL } \\
& \text { In BL }\end{array}$ & $\begin{array}{l}3 \\
3\end{array}$ & $\begin{array}{l}3 \\
2\end{array}$ & $\begin{array}{l}2 \\
1\end{array}$ & $\begin{array}{l}\mathrm{m} / \mathrm{s} \\
\mathrm{m} / \mathrm{s}\end{array}$ \\
\hline Minimum wind measurement success rate ${ }^{\mathrm{D}}$ & 50 & 50 & 50 & $\%$ \\
\hline \multicolumn{5}{|c|}{ 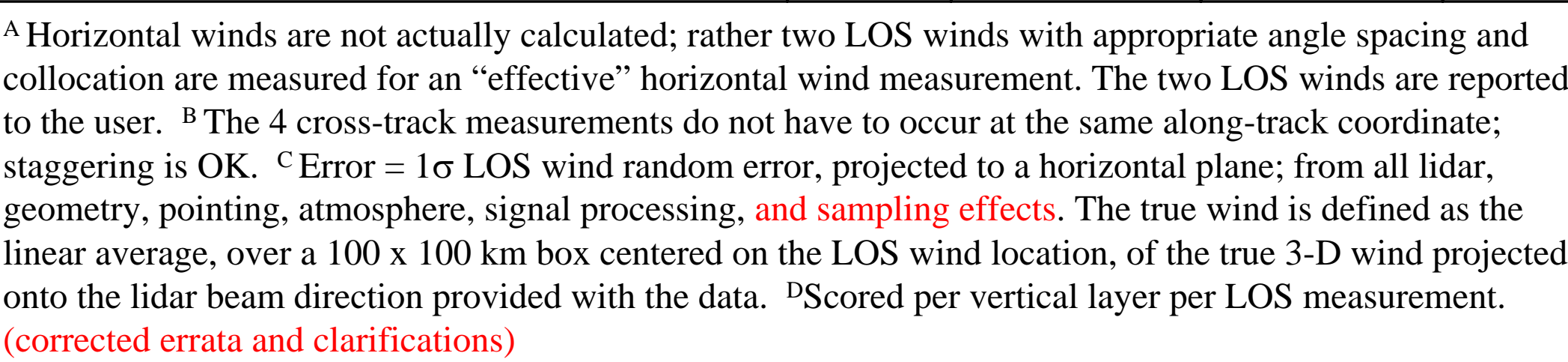 } \\
\hline
\end{tabular}




\section{Has The Gap Been Narrowed?}

\begin{tabular}{|c|c|}
\hline pre-1993 & $2007 *$ \\
\hline $\begin{array}{c}\text { 2-micron coherent lidar covers } \\
\text { entire troposphere }\end{array}$ & $\begin{array}{l}\text { 2-micron and 0.355-micron lidars } \\
\text { share the job - hybrid concept }\end{array}$ \\
\hline Required pulse energy: $20 \mathrm{~J}$ & Required pulse energy: $0.25 \mathrm{~J}$ \\
\hline Demonstrated pulse energy: $0.02 \mathrm{~J}$ & Demonstrated pulse energy: $1.2 \mathrm{~J}$ \\
\hline Factor of 1000 deficit $:$ & Factor of 5 margin $: ;$ \\
\hline
\end{tabular}

\section{Situation has improved by $37 \mathrm{~dB}$ !}

*2007: also advancements in crystal material, electrical efficiency, cooling method, component count, beam quality, pump laser diodes, and compact packaging 


\section{Summary and Conclusions}

- Combination of technology advances, hybrid Doppler lidar idea, and demonstration measurement requirements for the first mission has greatly narrowed the distance between demonstrated capability and needed mission performance

- Observing System Simulation Experiments have determined the required measurement performance

- Doppler lidar performance simulations, anchored with experimental results, have determined the required lidar parameters

- NASA's Instrument and Mission Design Teams have accommodated the lidar parameters in a notional mission

- There are numerous customers for the wind data

- The recent NRC Decadal Survey recommended the mission to NASA 


\section{Back Up Slides}

